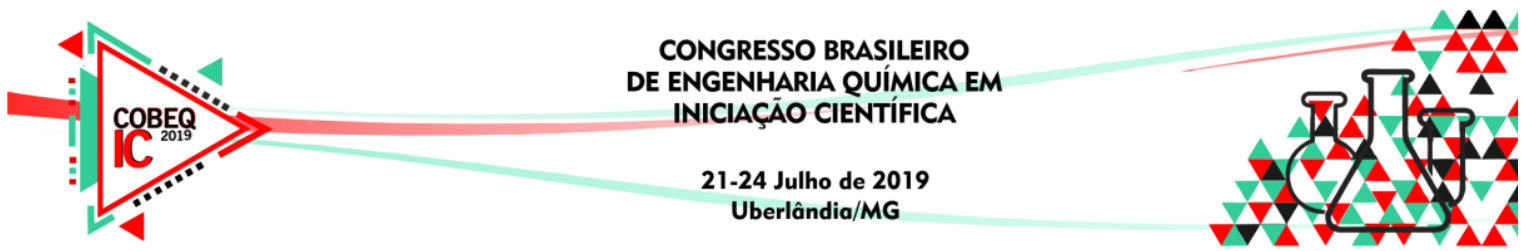

\title{
SENSORIAMENTO DE ÍNDICE DE REFRAÇÃO POR FIBRA ÓPTICA PLÁSTICA E NANOMATERIAIS
}

\author{
MARIANA R.P. VALE ${ }^{1}$, ISABEL C. S. CARVALHO ${ }^{2}$ e GREICE K.B. COSTA ${ }^{3}$ \\ ${ }^{1}$ Universidade Federal Rural do Rio de Janeiro, Departamento de Engenharia Química \\ ${ }^{2}$ Pontíficia Universidade Católica do Rio de Janeiro, Departamento de Física \\ ${ }^{3}$ Universidade Federal Rural do Rio de Janeiro, Departamento de Física \\ E-mail para contato: marianarpvale@hotmail.com
}

RESUMO - Neste artigo mostra-se os resultados obtidos por um Sensor de Fibra Óptica (POF) com base em Ressonância de Plasma de Superfície Localizada (RPSL) com a ponta revestida com Nanopartículas de Ouro (AuNPs) produzidas pelo método de sementes. Testamos sua sensibilidade à mudança do índice de refração (IR). As AuNPs mostraram boa sensibilidade em torno do IR da Escherichia Coli (1,33-1,39). A ponta da POF foi previamente preparada, retirando-se o revestimento com acetona e água destilada e, em seguida, foi imersa em solução de ciclopentanona para diminuir o núcleo para $700 \mu \mathrm{m}$ e, assim, atuar como lente acoplada. A solução de AuNPs foi caracterizada por microscopia eletrônica de transmissão (MET) e espectro de absorbância. As nanopartículas obtidas apresentaram $10 \mathrm{~nm}$ de diâmetro e o baricentro do espectro de absorção encontra-se em $526 \mathrm{~nm}$. A adesão à ponta POF foi investigada por Microscópio Eletrônico de Varredura com Canhão de Emissão de Campo (FEG-SEM), mostrando que o processo utilizado foi realizado com sucesso.

\section{INTRODUÇÃO}

Nanopartículas metálicas (1-100) nm em tamanho foram extensivamente pesquisadas na área de medicina para o diagnóstico de hipertensão arterial sistemática (Rolim et al., 2015), detecção de Bacillus anthracis (Hao et al., 2011), diagnóstico de câncer, terapia fototermal (Huang e El-Sayed, 2010) e dengue (Camara et al., 2013), devido à característica única dos metais nobres que depende fortemente do tamanho e da forma das nanopartículas. A Ressonância de Plasmon de Superfície Localizada (LSPR) é a oscilação coletiva ressonante de elétrons livres das nanopartículas metálicas sob irradiação de campo eletromagnético, sendo altamente sensível ao índice de refração (IR) do meio circundante, segundo Xiong e Lu (2015). As nanopartículas de ouro nos últimos anos tem sido o foco das aplicações nanobiológicas, como mencionado acima, por isso várias técnicas têm sido utilizadas para produzi-las, tais como evaporação térmica, ablação a laser e método de crescimento mediado por sementes. A coloração apresentada e a absorção da solução coloidal de ouro está relacionada à razão de aspecto das nanopartículas. Como mostrado nos trabalhos de Liz-Marzàn (2004), Herget e Wriedt (2012), a cor pode ser vermelha, violeta, azul, marrom amarelada ou marrom e a absorção plasmônica pode ser entendida pela teoria de Mie. Para nanopartículas de ouro menores que o comprimento de onda (cerca de $25 \mathrm{~nm}$ ) da luz absorvente, o coeficiente de extinção $\mathrm{K}$, representa o somatório de toda contribuição multipolar da oscilação elétrica e magnética para a absorção e dispersão do campo eletromagnético incidente, equação 1.

$$
K=\frac{18 \pi N V \varepsilon_{m}^{3 / 2}}{\lambda} \frac{\varepsilon^{2}}{\left[\varepsilon_{1}+2 \varepsilon_{m}\right]^{2}+\varepsilon_{2}^{2}}
$$




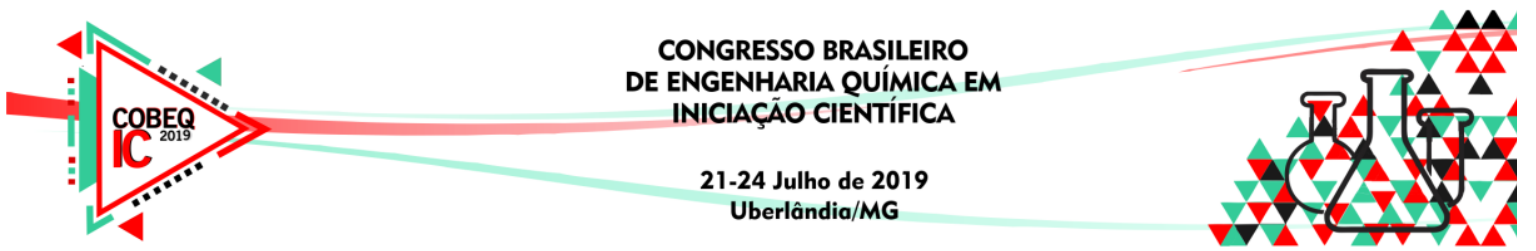

Onde, $\mathrm{N}$ é o número de partículas de volume $\mathrm{V}, \lambda$ é o comprimento de onda da radiação absorvente, $\varepsilon \mathrm{m}$ é a constante dielétrica do meio circundante e $\varepsilon 1$ e $\varepsilon 2$ são as partes reais e imaginárias da função dielétrica do material, respectivamente.

Levando em consideração a teoria de Mie que mostrou a dependência da absorção de plasmon com o tamanho das nanopartículas de ouro e o índice de refração circundante, produzimos nanoesferas de ouro de $10 \mathrm{~nm}$ de diâmetro para verificar sua sensibilidade à mudança de IR envolvente para posterior aplicação como sensor Escherichia Coli (IR = 1,331,39) (Wandermur et al., 2014).

\section{METODOLOGIA}

Nesta seção será mostrada a produção de nanoesferas de ouro, os procedimentos adotados para revestir a ponta da Fibra Ótica Plástica com estas e as medidas feitas para verificar a sensibilidade do sensor à mudanças no IR para futuras aplicações como sensor de Escherichia-Coli.

\subsection{Síntese de Nanoesferas (AuNPs)}

Nanoesferas de ouro foram produzidas com reagentes adquiridos no Sigma Aldrich, todos com pureza $\geq 99 \%$ e água deionizada com resistividade de $18 \mathrm{M} \Omega * \mathrm{~cm}$. Como mostrado no trabalho de Fontana (2011), a produção de nanoesferas de ouro consiste na preparação da semente e solução de crescimento com as seguintes concentrações.

Solução sementes: solução de $\operatorname{HCTAB}(10 \mathrm{~mL}, 0,10 \mathrm{M}), \mathrm{HAuCl}_{4} .3 \mathrm{H}_{2} \mathrm{O}(25 \mu \mathrm{L}, 0,10 \mathrm{M})$ e $\mathrm{NaBH}_{4}(600 \mu \mathrm{L}$ 0,01 M).

Solução de crescimento: solução de $\mathrm{HCTAB}(100 \mathrm{~mL}, 0,10 \mathrm{M}), \mathrm{HAuCl}_{4} .3 \mathrm{H}_{2} \mathrm{O}(500 \mu \mathrm{L}, 0,10$ $\mathrm{M}), \mathrm{AgNO}_{3}(80 \mu \mathrm{L} 0,10 \mathrm{M})$ e ácido ascórbico $(550 \mu \mathrm{L}, 0,10 \mathrm{M})$.

Apenas $100 \mu \mathrm{L}$ da solução de sementes foram utilizados na solução de crescimento, agitados por $1 \mathrm{~h}$ a $40^{\circ} \mathrm{C}$. A figura 1 mostra o método utilizado para caracterização das nanoesferas de ouro, obtido por meio de uma fração molar de 0,87 de Au. Na figura1a temos o espectro de absorbância com pico baricentivo a $526 \mathrm{~nm}$ e densidade óptica de 2,3, então 1,12 x $10^{13} \mathrm{~Np} / \mathrm{mL}$. Na figura $1 \mathrm{~b}$ mostra imagens de formas de nanoesferas de ouro, elas são monodispersas com $10 \mathrm{~nm}$ de diâmetro. Na figura 1c é possível ver os planos atômicos das nanoesferas de ouro. Na figura 1d mostra o padrão da difração de elétrons associada à fase $\left(\mathrm{NH}_{4}\right) 6\left(\mathrm{AuCl}_{4}\right) 3 \mathrm{Ag}_{2} \mathrm{Cl}_{5}$, sistema cristalino ortorrômbico e plano hkl (020), (301), (321), (611), (040), ( 602), (622), (042) e (912) identificados na imagem, preveniente da tabela JCPDS-PDF 70-0908 (JCPDS).

Figura 1 - (a)espectro de absorção; (b) Imagens de Microscópio Eletrônico de Transmissão; (c) Planos atômicos das nanoesferas de ouro e (d) Padrão de difração de elétrons da fase $\left(\mathrm{NH}_{4}\right) 6\left(\mathrm{AuCl}_{4}\right) 3 \mathrm{Ag}_{2} \mathrm{Cl}_{5}$

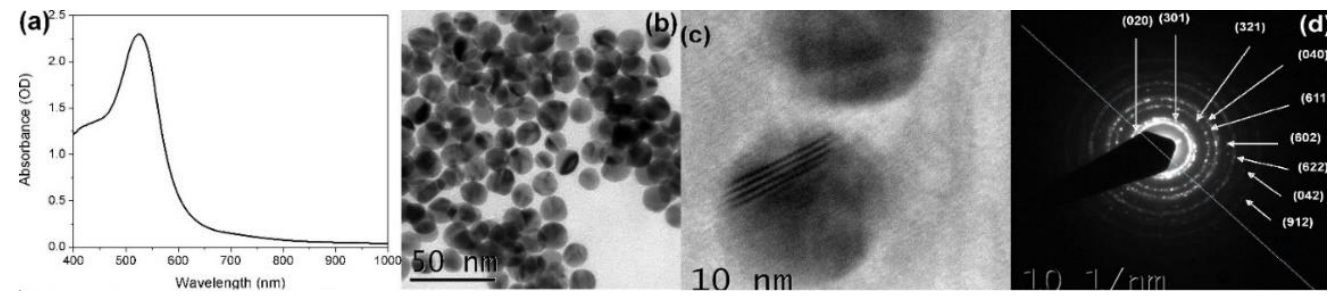

\subsection{Preparação da Ponta da POF}




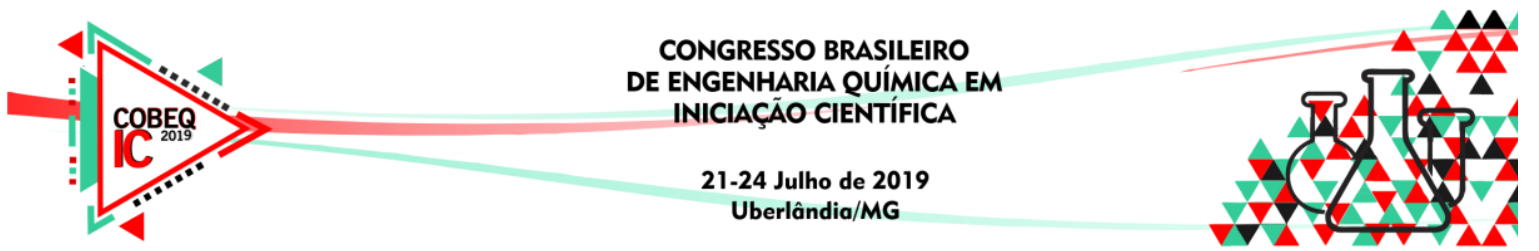

O POF utilizado é o CK-40 adquirido da indústria de fibra óptica ESKA com materiais de núcleo e revestimento de Resina de Polimetil-Metacrilato e Polímero Fluorado, respectivamente $\left(\mathrm{ESKA}^{\mathrm{TM}}\right)$. Foi previamente cortado em segmentos de $12 \mathrm{~cm}$ e o revestimento foi removido ao longo de $1 \mathrm{~cm}$ da extremidade da ponta usando quatro gotas de $100 \%$ de acetona no papel tóxico $(2 \times 2) \mathrm{cm}$ que foi imediatamente rotacionado e deslizou ao longo da região tratada por 15 segundos, após a adição de quatro gotas de água destilada e repetição do mesmo movimento na ponta POF para completar a remoção do revestimento. Após este passo, a região de tratamento foi limpa com água para neutralizar a solução (acetona-água) e também álcool isopropílico para remoção de graxas, como realizado no trabalho de Merchant et al. (1999). O último estágio é imergir na solução de ciclopentanona para diminuir o núcleo para $700 \mu \mathrm{m}$, cujo diâmetro é controlado pela evaporação do solvente, de modo a atuar como uma lente de curta distância focal (Xie et al. (2009). Na figura 2 é mostrado o processo de resultado descrito acima, (a) POF $(980 \mu \mathrm{m} / 1.000 \mu \mathrm{m})$ e (b) ponta POF com $700 \mu \mathrm{m}$.

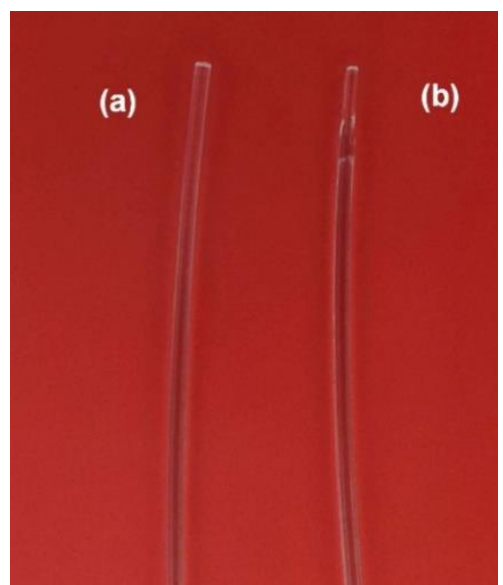

Figura 2 - (a) POF com $(980 \mu \mathrm{m} / 1.000 \mu \mathrm{m})$ e (b) ponta POF com $700 \mu \mathrm{m}$.

\subsection{Revestimento de Ponta POF com Nanoesferas de Ouro}

O revestimento da ponta da POF é realizado soltando a solução de ouro NPs ou imergindo a POF em Au NPs a $20^{\circ} \mathrm{C}$. De acordo com a secagem da solução, mais e mais NPs aderiam à ponta da POF, como mostrado por Zheng et al. (2008). Quando a solução de AuNPs secar na ponta da POF ocorre a nucleação dos AuNPs, cuja solução será aumentar a velocidade de evaporação da solução de AuNPs na ponta da POF, segundo Bigioni et al. (2006). Na figura 3 mostramos as imagens SEM da ponta da POF revestida com AuNPs: (a) $20 \mu \mathrm{L}$ da solução secando a 48h; (b) Duas gotas de $0,25 \mu \mathrm{L}$ foram implantadas no intervalo de 15 min para cada uma; (c) Dez gotas de 0,25 $\mu \mathrm{L}$ foram implantadas no intervalo de 15 min para cada uma; (d) Dez gotas de 0,25 $\mu \mathrm{L}$ na ponta POF, imagem SEM tire após as medidas de sensibilidade das nanoesferas de ouro para mudar o local do índice refrativo. 

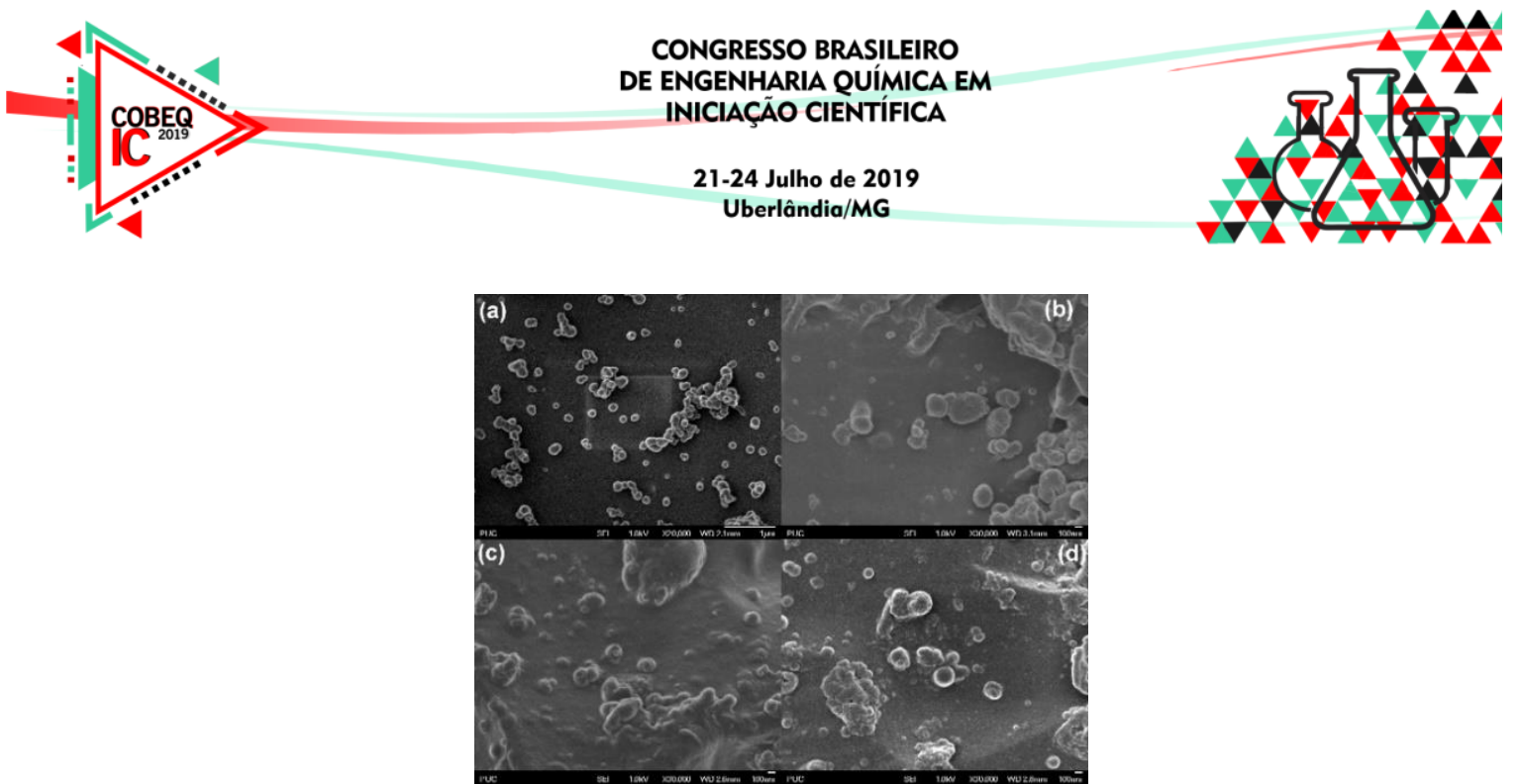

Figura 3 - (a) $20 \mu \mathrm{l}$ da solução secando a 48h, (b) Duas gotas de $0,25 \mu \mathrm{L}$ foram colocadas no intervalo de 15 min a cada uma, (c) Dez gotas de $0,25 \mu \mathrm{L}$ foram colocadas no intervalo de 15 min para cada um, e (d) Dez gotas de $0,25 \mu \mathrm{L}$ na ponta da POF, imagens SEM tiram após as medidas de sensibilidade das nanoesferas de ouro para mudar o local do índice refrativo.

Na figura 3 observa-se um aumento da nucleação dos AuNPs como resultado de um aumento da concentração dos AuNPs na ponta da POF (a) a (c), enquanto que em (d) os AuNPs permanecem na ponta da POF, mesmo após as medidas com índice refrativo e lavagem com água destilada.

\subsection{Medidas de Sensibilidade das Nanoesferas de Ouro à Mudança de IR}

A figura 4(a) mostra o conjunto utilizado para medições da sensibilidade de AuNPs a uma mudança no IR circundante com base na reflexão de Fresnel. As medições foram realizadas utilizando um espectrômetro Ocean Optics HR4000, um acoplador POF 2x1 com $1 \mathrm{~mm}$ de diâmetro, uma POF de $12 \mathrm{~cm}$ com a ponta revestida por nanoesferas de ouro, secando duas gotas de $0,25 \mu \mathrm{L}$ cada na ponta da POF. Na figura 4(b) é mostrado o espectro de absorção das AuNPs na ponta POF com pico LSPR em $541 \mathrm{~nm}$ e Densidade Óptica (DO) = 0,22. De acordo com a figura 3, mostrou a nucleação das nanoesferas de ouro, resultando neste espectro de grande largura de banda.
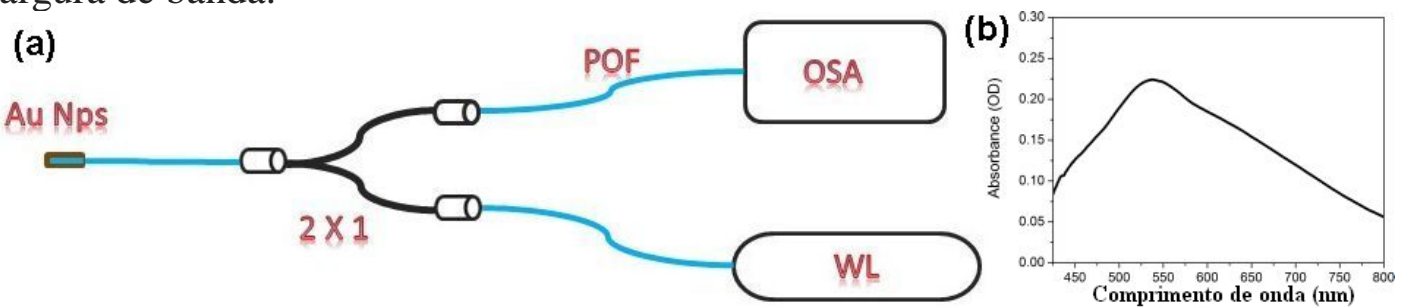

Fig. 4. (a) Configuração usada para medidas de sensibilidade das nanoesferas de ouro a uma mudança no índice de refração circundante. É composto por uma luz branca (WL) e um analisador de espectro óptico (OSA); (b) Espectro de absorção da ponta POF revestida com nanoesferas de ouro (duas gotas).

Na figura 5 mostramos as medidas de sensibilidade das nanoesferas de ouro ao IR. Aumentando o IR circundante de 1,0 para 1,7, observamos um desvio para o vermelho no pico do RPSL, porque o ângulo dos raios incidentes nos AuNPs depende fortemente do IR do meio circundante. $\mathrm{O}$ índice de refração líquido foi comprado nos laboratórios Car-gille com padrões $\mathrm{n}=1,26,1,30,1,40,1,48$ e 1,70 .

As AuNPs apresentam um vale de reflexão no ar em $541 \mathrm{~nm}$, quando imersos nesses líquidos consecutivamente, o LSPR deslocamento para $545 \mathrm{~nm}, 551 \mathrm{~nm}, 560 \mathrm{~nm}, 562 \mathrm{~nm}$ e $554 \mathrm{~nm}$, respectivamente. Analisando o LSPR das nanoesferas de ouro na ponta da POF com 


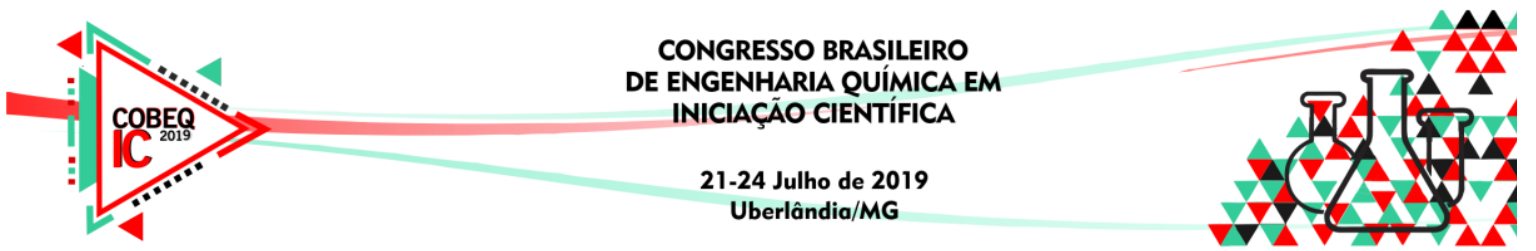

base na reflexão de Fresnel e sabendo que o índice de refração da POF é de 1,49. Nós temos que quando o índice do meio circundante se aproxima do índice de fibra, o sinal refletido é muito fraco, então foi necessário fazer um zoom neste sinal refletido em $\mathrm{n}=1.48$, que é mostrado no gráfico inserido na figura 5 (a). Quando $\mathrm{n}=1,7$, o índice de refração do meio externo é maior que o índice da fibra, então ocorre uma inversão de fase de $\pi$ radianos na onda de retorno (Kinoshita, 2013). As estruturas observadas em $732 \mathrm{~nm}$ nas bandas da figura 5(a) são devidas a impurezas da água destilada utilizada para lavar as nanoesferas de ouro na ponta POF após a imersão em cada líquido índice de refração. Na figura 5(b) temos a mudança de inclinação em função do índice de refração, mostrando uma boa sensibilidade das nanoesferas de ouro (90 nm / RIU) entre o RI 1,30-1,40.

(a)

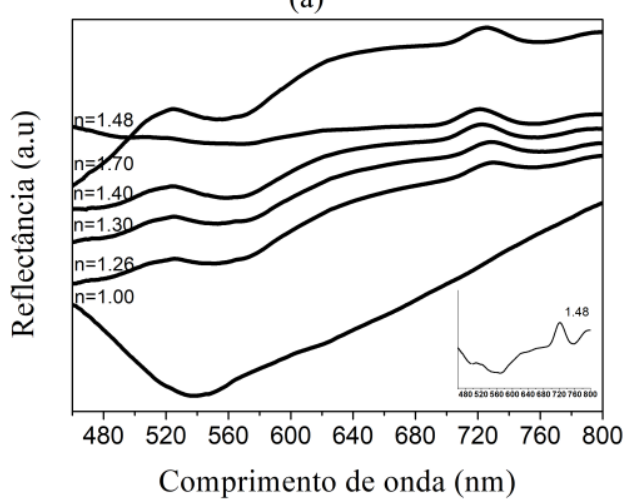

(b)

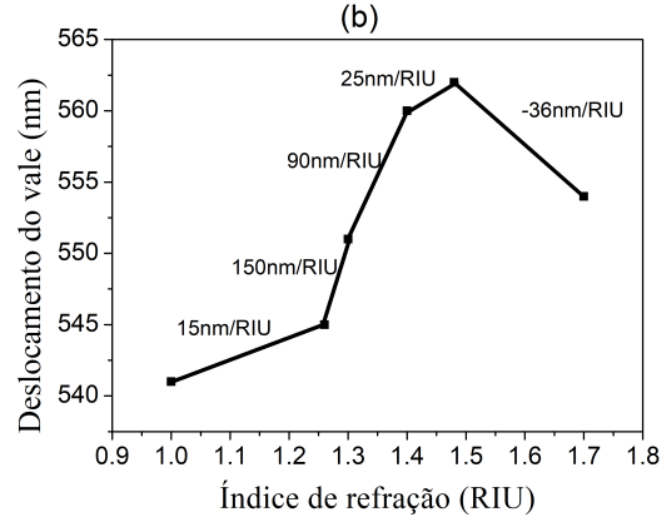

Figura 5 - (a) Luz refletida na ponta da POF revestida de nanoesferas de ouro com a mudança em torno do índice de refração de 1,0 a 1,7 e (b) Deslocamento do vale em função do índice de refração.

\section{CONCLUSÃO}

Um sensor de fibra óptica totalmente de plástico foi investigado quanto à sensibilidade ao índice de refração do meio. As nanoesferas de ouro foram utilizadas para revestir a ponta POF, as imagens TEM e o espectro de absorção mostraram que são monodispersas com $10 \mathrm{~nm}$ de diâmetro e baricentro em $526 \mathrm{~nm}$. Os procedimentos utilizados para diminuir o diâmetro da ponta da POF a $700 \mu \mathrm{m}$ para atuar como uma lente acoplada mostraram-se eficientes.

O protocolo de imobilização das nanoesferas de ouro na ponta da POF por secagem foi realizado, mas ocorreram nucleações das AuNPs, mostradas nas imagens MEV-FEG. A pesquisa da sensibilidade do sensor ao IR mostraram resultados promissores por medidas de reflexão de Fresnel baseadas na LSPR das AuNPs. Mostrou-se um desvio para o vermelho do vale do sinal refletido e exibiu uma boa sensibilidade de $90 \mathrm{~nm} /$ RIU entre 1,30-1,40, sendo este o meio que pretendemos trabalhar para sensoriar Escherichia-coli (1.33-1.39). Os próximos passos desta pesquisa são verificação da melhor sensibilidade do sensor POF, diminuindo o diâmetro da ponta POF, aumentando a velocidade de evaporação das gotas da solução de nanoesferas de ouro, a fim de reduzir a nucleação. Finalmente, pretende-se usar o sensor todo à fibra óptica plástica para detectar e quantificar Escherichia-coli.

\section{AGRADECIMENTOS}

Os autores agradecem ao CNPq pelo suporte financeiro, Laboratório de Optoeletrônica (LOpEL) da PUC-Rio e Laboratório de Nanomateriais e Fotônica da UFRRJ pelo suporte para o desenvolvimento da pesquisa. 


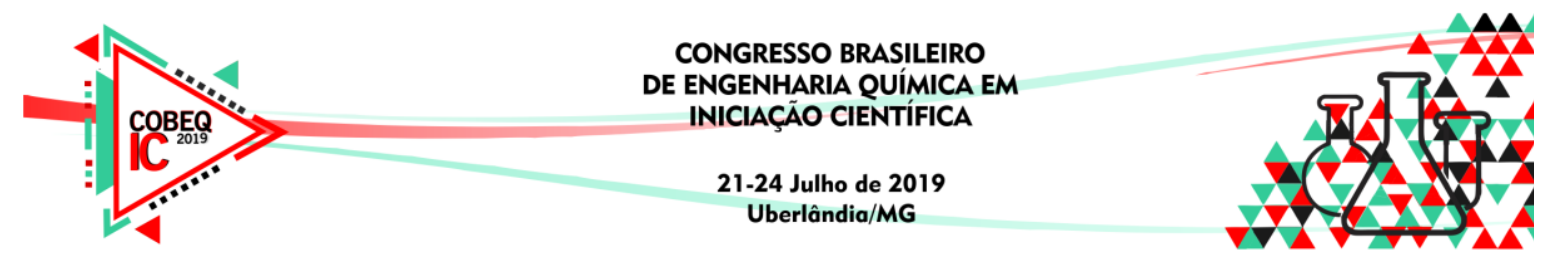

\section{REFERÊNCIAS}

BIGIONI, T. P.; LIN, X-M.; NGUYEN, T. T.; CORWIN, E. I.; WITTEN, T. A. e JAEGER, H. M. Kinetically driven self assembly of highly ordered nanoparticle monolayers. Letters 5, 265270 (2006).

CAMARA, A. R.; GOUVÊA, P. M. P.; DIAS, A. C. M. S.; BRAGA, A. M. B.; DUTRA, R. F.;ARAUJO, R.E. E CARVALHO,I.C.S.Dengue immunoassay with an LSPR fiber optic sens or. Optics Express 21(22), 27023-27031 (2013).

ESKA $^{\mathrm{TM}}$, Specification Sheet $C K-40$. Mitsubishi Rayon CO., LTD. DPF0831-15, 1-3 (2011). FONTANA, J.P. Self-Assembly and characterization of anisotropic metamaterials. Kent State University (2011).

HAO, R-Z. ; SONG, H-B. ; ZUO, G-M.; YANG, R-F. ;WEI, H-P. ; WANG, D-B. ; CUI, Z-Q. ; ZHANG, Z. ;CHENG, Z-X. e ZHANG, X-Z. DNA probe functionalized QCM biosensor based on gold nanoparticles amplification for Bacillus anthracis detection. Biosensors and Bioelectronics 26, 3398-3404 (2011).

HERGET, W.; WRIEDT, T. The Mie theory: Basic and applications. Springer 169, 259p. (2012).

HUANG, X. e EL-SAYED, M. A. Gold nanoparticle: Optical properties and implementations in cancer diagnosis and photothermal therapy. Journal and advanced research 1, 13-28 (2010).

JCPDS-International Centre for Diffraction Data.

KINOSHITA, S. Pattern Formations and oscillatory phenomena. Elsevier, 263p. (2013).

LIZ-MARZÁN, L. M. Nanometals: Formation and color. Elsevier Ltd, 26-31 (2004).

MERCHANT, D.F.; SCULLY, P.J. E SCHMITT, N.F. Chemical tapering of polymer optical fiber. Sensors and Actuators 76, 365-371 (1999).

ROLIM, T.; CANCINO, J; ZUCOLOTTO, V. A nanostructured genosensor for the early diagnosis of systemic arterial hypertension, Biomed macrodevices, 1-9 (2015).

WANDERMUR, G.; RODRIGUES, D.; ALLIL, R.; QUEIROZ, V.; PEIXOTO, R.; WERNECK, M. E MIGUEL, M. Plastic optical fiber-based biosensor platform for rapid cell detection. Biosensor and Bioelectronics 54, 661-666 (2014).

XIE, Z.; TAO, J.; LU, Y.; LIN, K.; YAN, J.; WANG, P. EeMING, H. Polymer optical fiber SERS sensor with gold nanorods. Optics communications 282, 439-442 (2009).

XIONG, Y. e LU, X. Metallic nanostructures. Springer, 301p (2015).

ZHENG, R-S. ; LU, Y-H. ; CHIE, Z-G. ; TAO, J. ; LIN, K-Q. e MING, H. Surface Plasmon Resonance sensors based on polymer optical fiber. Journal of electronic science and technology of china 6(4), 357-360 (2008). 